\title{
Spectral Counting of Triangles in Power-Law Networks via Element-Wise Sparsification
}

\author{
Charalampos E. Tsourakakis \\ School of Computer Science \\ Carnegie Mellon University \\ 5000 Forbes Avenue, Pittsburgh, PA 15213-3891 \\ ctsourak@cs.cmu.edu \\ Eirinaios Michelakis \\ EECS University of California, Berkeley \\ 387 Soda Hall, Berkeley, CA 94720-1776 \\ ireneos@cs.berkeley.edu
}

\author{
Petros Drineas \\ School of Computer Science \\ Rensselaer Polytechnic Institute \\ 110 8th Street, Troy, NY 12180-3590 \\ drinep@cs.rpi.edu \\ Ioannis Koutis \\ School of Computer Science \\ Carnegie Mellon University \\ 5000 Forbes Avenue, Pittsburgh, PA 15213-3891 \\ jkoutis@cs.cmu.edu
}

\author{
Christos Faloutsos \\ School of Computer Science \\ Carnegie Mellon University \\ 5000 Forbes Avenue, Pittsburgh, PA 15213-3891 \\ christos@cs.cmu.edu
}

\begin{abstract}
Triangle counting is an important problem in graph mining. The clustering coefficient and the transitivity ratio, two commonly used measures effectively quantify the triangle density in order to quantify the fact that friends of friends tend to be friends themselves. Furthermore, several successful graph mining applications rely on the number of triangles.

In this paper, we study the problem of counting triangles in large, power-law networks. Our algorithm, SPARSIFYINGEIGENTRIANGLE, relies on the spectral properties of power-law networks and the Achlioptas-McSherry sparsification process. SPARSIFYINGEIGENTRIANGLE is easy to parallelize, fast and accurate.

We verify the validity of our approach with several experiments in real-world graphs, where we achieve at the same time high accuracy and important speedup versus a straight-forward exact counting competitor.
\end{abstract}

\section{Introduction}

It is a well known fact in social network analysis that friends of friends tend to be friends themselves [23]. Tri- angles are a main indicator of this property. Two measures that quantify the triangle density of a graph are the clustering coefficient and the transitivity ratio ([19]).

Besides the significance of triangles in network analysis statistics, they also play an important role in graph mining applications: Eckmann and Moses showed how one can use triangles in order to uncover the hidden thematic structure of the web [11] and Beccheti et al. in [5] used the local distribution of triangles and the clustering coefficient to detect spamming activity. Furthermore, triangle-related power laws [21] can be used to define outliers in a graph with respect to triangles.

In this paper we focus on the problem of counting triangles in large networks. The main contribution of this work is a novel method for counting triangles in a large powerlaw network: we show how one can sparsify the graph converting it into another weighted graph, with significantly smaller number of edges and counting the number of triangles in that one with a recently introduced method [21], called EigenTriangle. Furthermore, our method is easy to parallelize since it uses only matrix-vector multiplications, easy to implement and most importantly gives significant speedups versus a straight-forward competitor. Finally, we validate the validity of our approach in several real world networks, where we achieve important speedups 


\begin{tabular}{ll}
\hline Sym. & Definition \\
\hline$G$ & Undirected simple graph \\
$d_{\max }$ & maximum node degree \\
$\Delta$ & total number of triangles \\
$\Delta^{\prime}$ & EIGENTRIANGLE's estima- \\
& tion of $\Delta$ \\
$m, n$ & Number of edges and nodes. \\
{$[n]=(1 . . n)$} & Node ids \\
$\mathbf{A}$ & Adjacency matrix \\
$\lambda_{i}$ & top- $i$-th eigenvalue (absolute \\
$\overrightarrow{\Lambda_{k}}=\left[\lambda_{i}\right]_{i=1 . . k}$ & value) \\
$p$ & $k$ top eigenvalues \\
& sparsification parameter \\
& (probability of keeping an \\
& edge) \\
\hline
\end{tabular}

Table 1. Definitions of symbols and acronyms

while being very accurate.

The outline of the paper is as follows: in section 2 we present briefly the related work, in section 3 we describe the proposed algorithm and in section 4 we show the experimental results. We conclude in section 5 .

\section{Background and Related Work}

In this section we describe briefly existing work on the problem of counting triangles and the Achlioptas-McSherry low rank approximation algorithm. In the rest of the paper, we use the symbols described in Table 1.

Let $G(V, E), \mathrm{n}=|V|, \mathrm{m}=|E|$ be an undirected graph without self-edges. A triangle is defined as a three node fully connected subgraph of $G$.

Exact Counting Methods The obvious way to count the number of triangles in a graph is to examine each of the $\left(\begin{array}{l}n \\ 3\end{array}\right)$ combinations of nodes and check whether they form a triangle or not. As the procedure suggests, the time complexity is $O\left(n^{3}\right)$.

Since the problem of counting triangles can be reduced to matrix multiplication, the complexity of counting triangles can be reduced as well to $O\left(n^{2.376}\right)$ [8]. This is also the lowest time complexity. Alon, Yuster and Zwick in [3] gave an algorithm of $O\left(m^{\frac{2 \omega}{\omega+1}}\right) \subset O\left(m^{1.41}\right)$ time complexity and of $\Theta\left(n^{2}\right)$ space complexity. However, these methods suffer from $\Theta\left(n^{2}\right)$ space complexity.

Therefore, listing methods ([20]) are preferred against matrix-multiplication based methods. Such methods are the NodeIterator and the EdgeIterator The NodeItERATOR considers each one of the $n$ nodes and examines which pairs of its neighbors are connected. The time complexity of the NODEITERATOR is $O\left(n d_{\max }^{2}\right)$. This is a significant improvement over the brute-force approach when the graph is sparse. The EDGEITERATOR algorithm computes for each edge the number of triangles that contain it. The time complexity of this algorithm is $O\left(m d_{\max }\right)$. Both methods are equivalent asymptotically ([20]). Schank and Wagner in [20] propose the forward algorithm with running time $\Theta\left(m^{\frac{3}{2}}\right)$ and space complexity $O(m)$. A nice survey and the state-of-the-art algorithms are described in [17].

Streaming Algorithms In the streaming approach, we restrict ourselves to one or at most a constant number of passes over the data. The goal it to output an accurate estimate of the number of triangles with high probability. Z.Bar-Yossef, Kumar and Sivakumar showed in [4] how one can approximate the number of triangles by using the AlonMatias-Szegedy ([2]) method for approximating frequency moments. New streaming algorithms were introduced in [6].

Semi-streaming model Recently, Becchetti, Boldi, Castillo and Gionis introduced the semi-streaming model in [5] to solve the local triangle counting problem. Their method relies on the locality sensitivity hashing concept. In contrast to the streaming model, this model relaxes the strict restriction of the constant number of passes over the data. Instead it performs $O(\log (n))$ sequential scans over the edge file.

EigenTriangle Recently, Tsourakakis gave two approximation algorithms in [21] for counting the total number of triangles and the triangles per node. It was observed that a low-rank approximation of the adjacency matrix yields in many real-world networks a fast, accurate and parallelizable method for counting triangles in power-law networks. The first theorem in [21] which is of interest to us in this work is the following:

$$
\Delta(G)=\frac{1}{6} \sum_{i=1}^{n} \lambda_{i}^{3}
$$

Achlioptas-McSherry Low Rank Approximation Algorithm Approximating a matrix with a low rank matrix is a task occuring frequently desired task in many applications ( e.g. [22],[9]) Since computing the optimal solution is expensive, often approximate solutions are used in practice (e.g [10] and [14]). Achlioptas and McSherry showed in [1] how one interested in a low rank approximation of a matrix $A$ can get a matrix $\hat{A}$ that has the following properties.

$$
\left\|A-\hat{A}^{(k)}\right\|_{2} \leq\left\|A-A^{(k)}\right\|_{2}+O\left(\left(\frac{n}{p}\right)^{\frac{1}{2}}\right)
$$




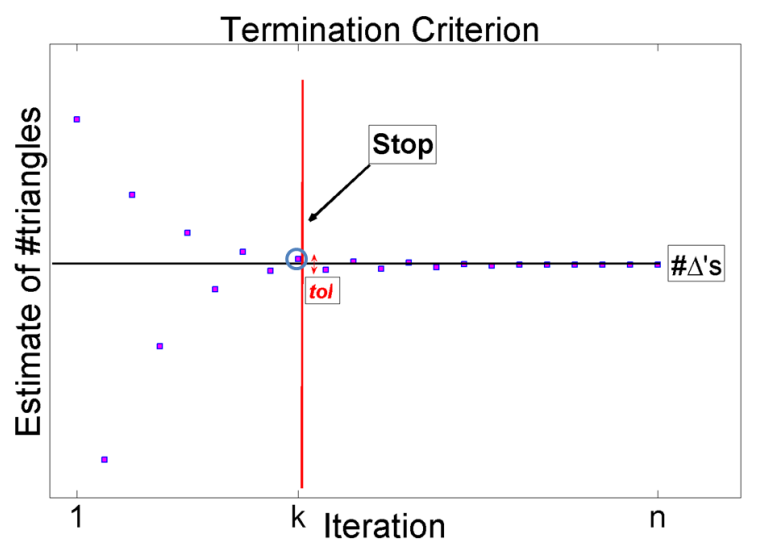

Figure 1. Termination criterion for SPARSIFYINGEIGENTRIANGLE. Plots the estimate of triangles at the $i$-th iteration vs. $i$. The algorithm decides at the $k$-th iteration to stop since its estimate did not change significantly from the $k$-th to the $(k+1)$-th iteration. As we see from the plot, the encircled estimate is very close to the actual value of the \#triangles

$$
\left\|A-\hat{A}^{(k)}\right\|_{F} \leq\left\|A-A^{(k)}\right\|_{F}+O\left(\left(\frac{n}{p}\right)^{\frac{1}{4}}\left\|\hat{A}^{(k)}\right\|_{F}^{\frac{1}{2}}\right.
$$

These equation reveal the existence of a matrix $\hat{A}^{(k)}$ for a given $k$ "close" to the optimal $A^{(k)}$ with respect to both the 2-norm and the Frobenius norm. Furthermore, they showed -among other things as well- how one can obtain the $\hat{A}$ in a very simple way: Toss a biased coin for each entry $(i, j)$ of the matrix $A$ with probability $p$ of keeping that specific entry. In case we keep the entry $(i, j)$, then $\hat{A}(i, j)$ becomes equal to $\frac{A_{i j}}{p}$.

\section{Proposed Method}

Our method builds on the top of the EIgENTRIANGLE and Achlioptas-McSherry algorithms' ideas. These are the following: (a) A low rank approximation of the adjacency matrix gives a good estimate of the number of triangles in the graph. (b) We can keep a small percentage of the total edges of the graph and keep the top eigenvalues of the sparsified graph very close to the ones of the initial graph.

Our algorithm, SPARSIFYINGEIGENTRIANGLE, takes two parameters, the tol parameter and the sparsification parameter $p$, as can be seen from the pseudocode. As we see the algorithm works in two stages. First it performs one pass over the edges of the graph (non-zero entries of the adjacency matrix). For each edge we toss a biased coin

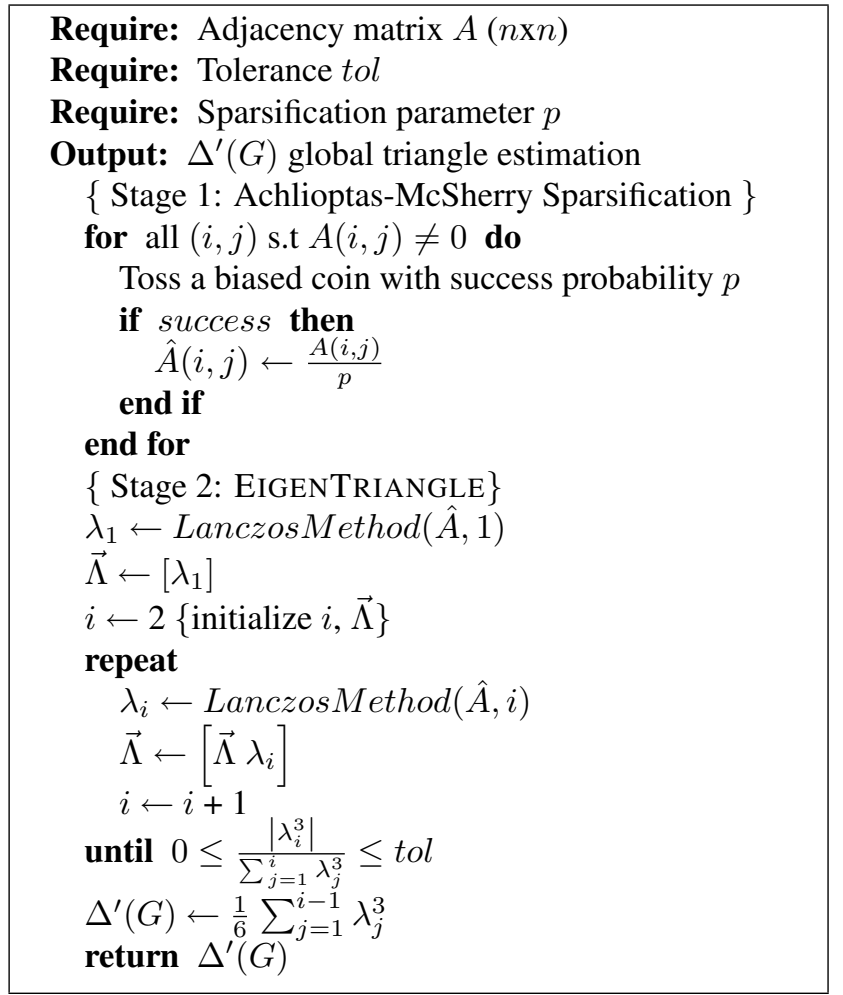

Algorithm 1: The SPARSIFYINGEIGENTRIANGLE algorithm

with probability $p$ of keeping an edge. In case we keep the edge, then we attach a weight of $\frac{1}{p}$ to that edge. Therefore, in expectation, at the end of the pass, we keep pm edges in total, all of them weighted with value equal to $\frac{1}{p}$. After the sparsification stage, the algorithm moves into the EIGENTRIANGLE stage. In this phase, we perform an iterative eigen-computation of the sparse adjacency matrix $\hat{A}$ until we observe that the cube of the absolute value of the eigenvalue being computed is significantly smaller than the estimate of triangles made until then. This is exactly the intuition behind the tol parameter: stop iterating when the eigenvalue just computed does not contribute significantly to the estimate. The intuition behind this stopping criterion is shown in figure 1.

The algorithm used in the eigen-computation is Lanczos method an efficient method for finding the top eigenvalues in sparse, symmetric matrices. Golub and Van Loan in [15] provide an excellent treatment of Lanczos method. One of the important properties of Lanczos is that the number of passes the edges depends on the spectral gaps of the eigenvalues to be computed. In our case due to the powerlaw that holds for the top-eigenvalues [12], [18],[7] Lanczos converges fast ([16]).

When the iteration stops, the algorithm outputs the estimate of the number of triangles in the graph as the sum 


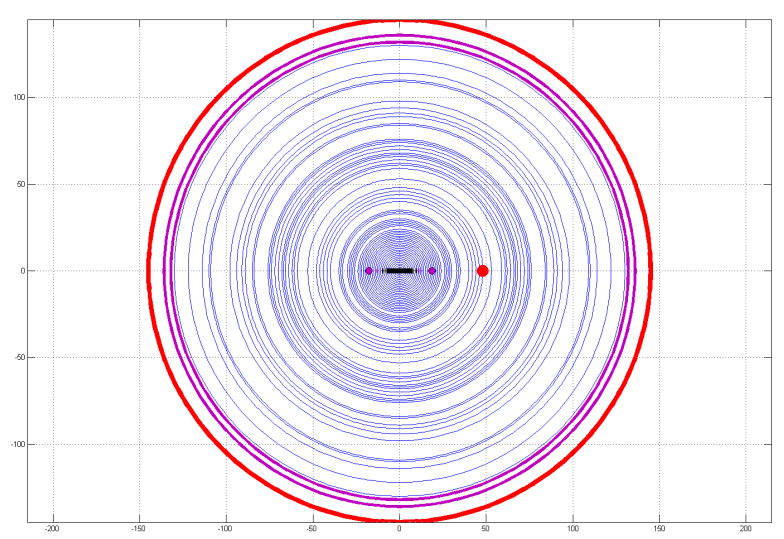

Figure 2. Gershgorin circles for a small graph (airports) on the complex plane. As we see from the plot, all eigenvalues lie on the real axis due to the symmetry of the adjacency matrix, most of them are almost symmetric around zero, and a few ones are detached from the rest. The top eigenvalue is denoted with red, the second and third with purple and so are the corresponding circles.

of the cubes of the computed eigenvalues divided by 6 , in accordance to the EIGENTRIANGLEtheorem ([21]).

Our algorithm, SPARSIFYINGEIGENTRIANGLE works for many real-world networks very fast in practice, due to the following properties:

1. Top eigenvalues follow a power law which implies the following desired properties:

- Few eigenvalues contribute a lot to the number of triangles.

- Cubes amplify this even more.

- Lanczos converges very fast.

2. The rest of the eigenvalues are almost symmetric around zero, and therefore they can be discarded since the sum of their cubes will not contribute significantly to the number of triangles.

This properties are illustrated in figure 2 where we see the Gershgorin circles which are simple upper bounds on the eigenvalues and the actual eigenvalues, and are in accordance with the observations made by Farkas, Derenyi, Barabasi and Vicsek in [13].

\section{Experiments}

The graphs we used in our experiments are described in Table 2. We implemented all our algorithms in MATLAB and the experiments ran on a 4 GB RAM, Intel(R)
Core(TM)2 Duo CPU at 2.4GHz Windows Vista machine. We report the results of our method in terms of the speedup vs. the NODEITERATOR.

\begin{tabular}{r|r|l} 
Nodes & Edges & Description \\
\hline \hline 404,733 & $2,110,078$ & Flickr \\
\hline 13,332 & 148,038 & $\begin{array}{l}\text { Reuters news, } \\
\text { Sept 9-11,2001. }\end{array}$ \\
\hline 13,579 & 37,448 & AS Oregon \\
\hline 23,389 & 47,448 & CAIDA AS \\
\hline
\end{tabular}

Table 2. Order and size of networks used.

In order to avoid running into dilemmas for the choice of the tolerance parameter, we adapt the empirical rule-ofthumb from [21], where it was observed in a wide range of experiments that a) typically a 6.2 rank approximation per average is good enough to acquire more than $95 \%$ accuracy and $b$ ) the maximum number of eigenvalues needed was 23 . Therefore, in our experiments we compute for each graph the top-30 eigenvalues, even if less eigenvalues can provide an accurate estimation. The results are shown in figure 3. For each dataset, we plot the accuracy and the speedup vs. the NODEITERATOR for the esimation resulting after computing one to thirty top eigenvalues. Similar results are obtained for other graphs of about the same size as well, which are omitted here due to the limited space. The plots presented are representative of what can see when somebody runs SPARSIFYINGEIGENTRIANGLE (e.g., different scenarios that can occur in the convergence of the estimate towards the real value).

These plots reveal the following facts: 1) Even when we keep via the Achlioptas-McSherry sparsification a small percentage e.g $10 \%$ of the graph edges the eigenvalues remain very close to the real ones. 2) Few top eigenvalues are enough to get a good estimate of the total number of triangles in the graph. 3) Speedups, even for graphs with few tenths of thousand or few million edges are important. 4) The expected trend of significant savings as the number of non-zeros elements of the matrix gets smaller is not clearly observed and this is due to the implementation properties of MATLAB's eigensolver. However, this this phenomenon will be eliminated when our algorithm is applied to larger graphs.

\section{Conclusions}

In this follow-up work, we introduced the SPARSIFYINGEIGENTRIANGLE, a fast, parallelizable algorithm that can be used in cases where the graph of interest does not fit in the main memory. The main idea of the algorithm is to use a low-rank approximation of the matrix which is 
generated via the Achlioptas-McSherry ([1]) sparsification of the adjacency matrix to compute the number of triangles based on the EIGENTRIANGLE algorithm ([21]). Furthermore, we show that even when keeping $10 \%$ of the graph edges one can compute the number of triangles in a very accurate and fast way.

\section{References}

[1] D. Achlioptas and F. McSherry. Fast computation of low rank matrix approximation. In STOC, 2001.

[2] N. Alon, Y. Matias, and M. Szegedy. The space complexity of approximating the frequency moments. In STOC '96: Proceedings of the twenty-eighth annual ACM symposium on Theory of computing, pages 2029, New York, NY, USA, 1996. ACM.

[3] N. Alon, R. Yuster, and U. Zwick. Finding and counting given length cycles. Algorithmica, 17(3):209-223, 1997.

[4] Z. Bar-Yosseff, R. Kumar, and D. Sivakumar. Reductions in streaming algorithms, with an application to counting triangles in graphs. In SODA '02: Proceedings of the thirteenth annual ACM-SIAM symposium on Discrete algorithms, pages 623-632, Philadelphia, PA, USA, 2002. Society for Industrial and Applied Mathematics.

[5] L. Becchetti, P. Boldi, C. Castillo, and A. Gionis. Efficient semi-streaming algorithms for local triangle counting in massive graphs. In Proceedings of ACM KDD, Las Vegas, NV, USA, August 2008.

[6] L. S. Buriol, G. Frahling, S. Leonardi, A. MarchettiSpaccamela, and C. Sohler. Counting triangles in data streams. In PODS '06: Proceedings of the twentyfifth ACM SIGMOD-SIGACT-SIGART symposium on Principles of database systems, pages 253-262, New York, NY, USA, 2006. ACM.

[7] F. Chung, L. Lu, and V. Vu. Eigenvalues of random power law graphs. Annals of Combinatorics, 7(1):2133, June 2003.

[8] D. Coppersmith and S. Winograd. Matrix multiplication via arithmetic progressions. In STOC '87: Proceedings of the nineteenth annual ACM conference on Theory of computing, pages 1-6, New York, NY, USA, 1987. ACM.

[9] S. Deerwester, S. T. Dumais, G. W. Furnas, T. K. Landauer, and R. Harshman. Indexing by latent semantic analysis. Journal of the American Society for Information Science, 41:391-407, 1990.
[10] P. Drineas and R. Kannan. Pass efficient algorithms for approximating large matrices. In SODA '03: Proceedings of the fourteenth annual ACM-SIAM symposium on Discrete algorithms, pages 223-232, Philadelphia, PA, USA, 2003. Society for Industrial and Applied Mathematics.

[11] J.-P. Eckmann and E. Moses. Curvature of co-links uncovers hidden thematic layers in the world wide web. PNAS, 99(9):5825-5829, April 2002.

[12] M. Faloutsos, P. Faloutsos, and C. Faloutsos. On power-law relationships of the internet topology. In SIGCOMM, pages 251-262, 1999.

[13] I. J. Farkas, I. Derenyi, A.-L. Barabasi, and T. Vicsek. Spectra of "real-world" graphs: Beyond the semicircle law. Physical Review E, 64:1, 2001.

[14] A. Frieze, R. Kannan, and S. Vempala. Fast montecarlo algorithms for finding low-rank approximations. In In Proceedings of the 39th Annual IEEE Symposium on Foundations of Computer Science, pages 370-378, 1998.

[15] G. Golub and C. Van Loan. Matrix Computations. JohnsHopkinsPress, Baltimore, MD, second edition, 1989.

[16] D. J. Applied Numerical Linear Algebra. SIAM, Philadelphia, PA, 1997.

[17] M. Latapy. Main-memory triangle computations for very large (sparse (power-law)) graphs. Theor. Comput. Sci., 407(1-3):458-473, 2008.

[18] M. Mihail and C. Papadimitriou. the eigenvalue power law, 2002.

[19] M. E. J. Newman. The structure and function of complex networks. SIAM Review, 45:167-256, 2003.

[20] Thomas Schank and Dorothea Wagner. DELIS-TR0043 - finding, counting and listing all triangles in large graphs, an experimental study. techreport 0043, submitted, 2004.

[21] C. Tsourakakis. Fast counting of triangles in large real networks, without counting: Algorithms and laws. In ICDM, 2008.

[22] M. Turk and A. Pentland. Eigenfaces for recognition. Journal of Cognitive Neuroscience, 3(1):71-86, 1991.

[23] S. Wasserman and K. Faust. Social network analysis. Cambridge University Press, Cambridge, 1994. 


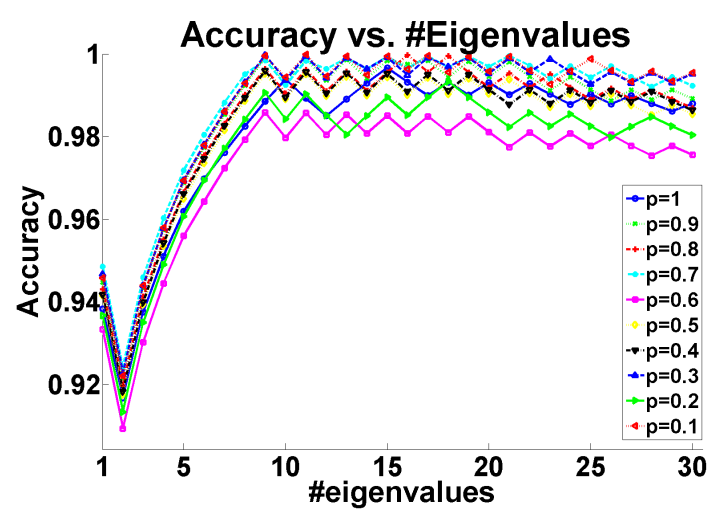

Accuracy vs. \#Eigenvalues

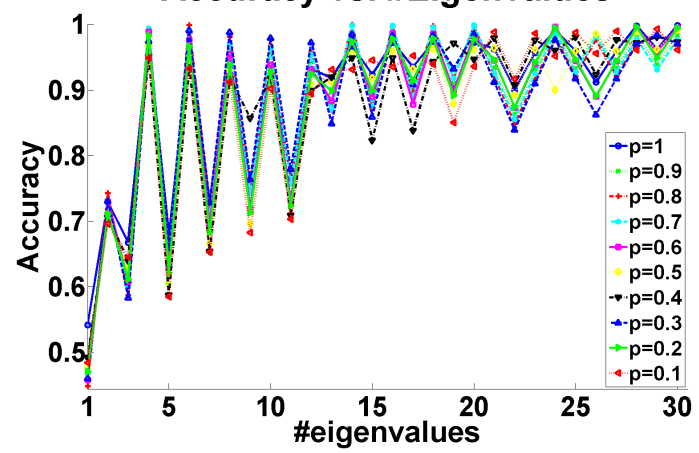

Accuracy vs. \#Eigenvalues

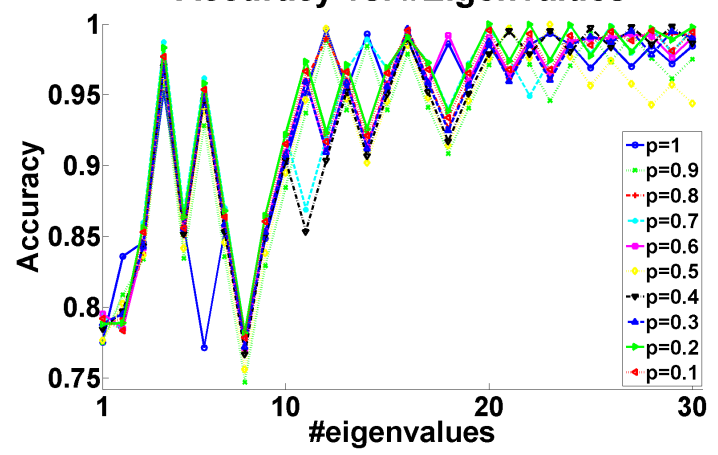

Accuracy vs. \#Eigenvalues

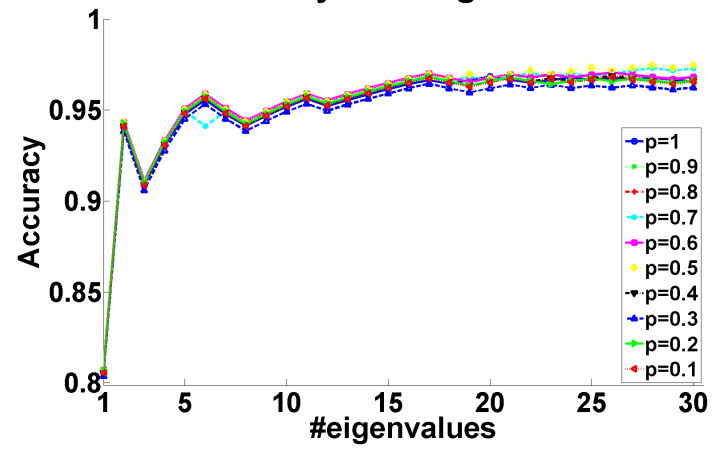

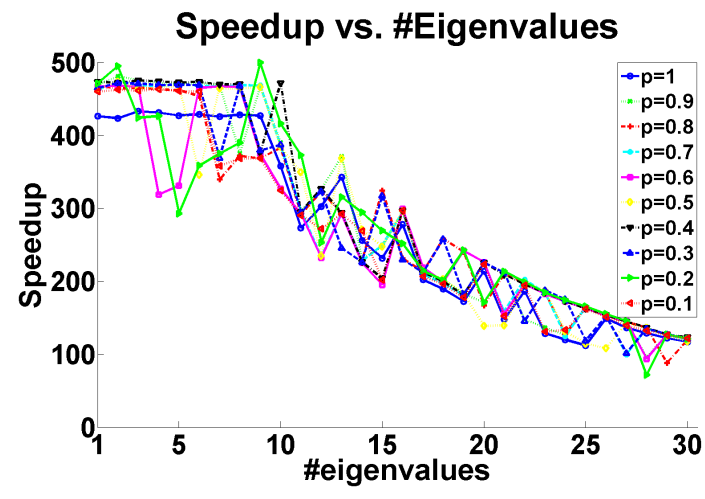

Speedup vs. \#Eigenvalues

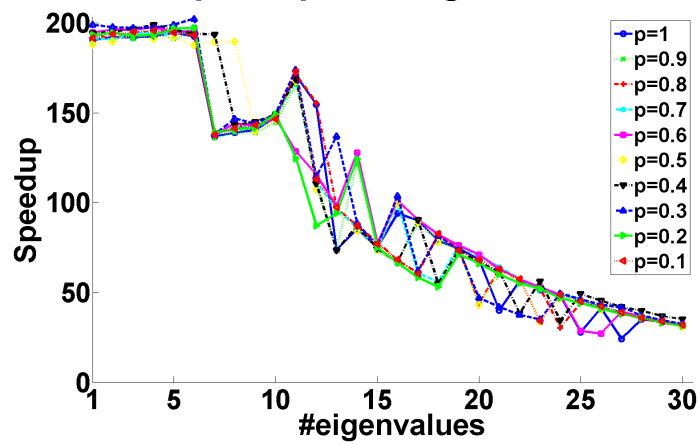

Speedup vs. \#Eigenvalues

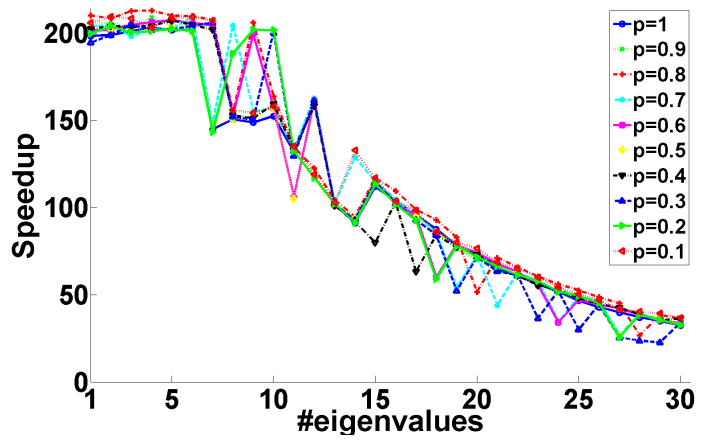

Speedup vs. \#Eigenvalues

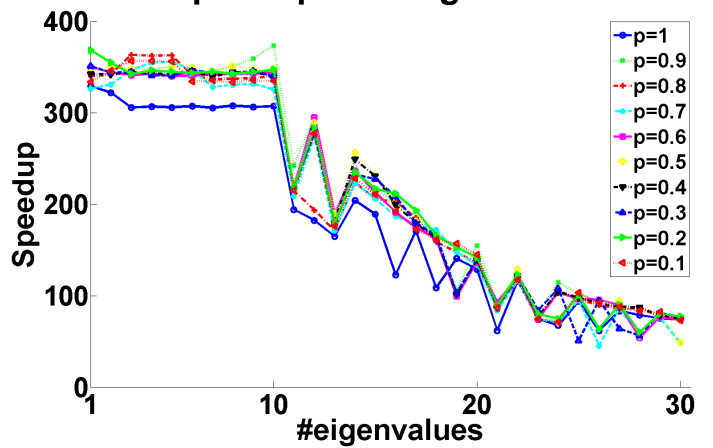

Figure 3. Experimental Results for four different datasets. (a) Reuters (b) AS CAIDA (c) AS Oregon (d) Flickr. Observe the following points: (1) the high accuracy obtained in the estimate after the top-5 eigenvalues, (2) the descending "oscillation" that moves towards the true value in different ways (3) the important speedups that we obtain while being accurate. 